\title{
Postsurgical Stage I Hepatoblastoma
}

National Cancer Institute

\section{Source}

National Cancer Institute. Postsurgical Stage I Hepatoblastoma. NCI Thesaurus. Code C7146.

No metastases, tumor completely resected. (from PDQ 2004) 